\title{
Article \\ Performance Enhancement of Inexpensive Glow Discharge Detector Operating in Up-Conversion Mode in Millimeter Wave Detection for Focal Plane Arrays
}

\author{
Arun Ramachandra Kurup ${ }^{1, *(\mathbb{D}}$, Daniel Rozban ${ }^{1}$, Lidor Kahana ${ }^{1}\left(\mathbb{D}\right.$, Amir Abramovich ${ }^{1}\left(\mathbb{D}\right.$, Yitzhak Yitzhaky ${ }^{2}$ (D) \\ and Natan Kopeika ${ }^{2}$ (D) \\ 1 Department of Electrical and Electronic Engineering, Ariel University, Ariel 40700, Israel; \\ rozbandaniel@ariel.ac.il (D.R.); lidor8531@gmail.com (L.K.); amir007@ariel.ac.il (A.A.) \\ 2 Department of Electro-Optics and Photonics Engineering, School of Electrical and Computer Engineering, \\ Ben-Gurion University of the Negev, Beer Sheva 84105, Israel; ytshak@bgu.ac.il (Y.Y.); \\ kopeika@bgu.ac.il (N.K.) \\ * Correspondence: arunra@ariel.ac.il
}

check for updates

Citation: Ramachandra Kurup, A.; Rozban, D.; Kahana, L.; Abramovich, A.; Yitzhaky, Y.; Kopeika, N.

Performance Enhancement of Inexpensive Glow Discharge Detector Operating in Up-Conversion Mode in Millimeter Wave Detection for Focal Plane Arrays. Appl. Sci. 2021, 11, 9564 https://doi.org/10.3390/app11209564

Academic Editor: Yiming Zhu

Received: 15 August 2021

Accepted: 12 October 2021

Published: 14 October 2021

Publisher's Note: MDPI stays neutral with regard to jurisdictional claims in published maps and institutional affiliations.

Copyright: (c) 2021 by the authors. Licensee MDPI, Basel, Switzerland. This article is an open access article distributed under the terms and conditions of the Creative Commons Attribution (CC BY) license (https:/ / creativecommons.org/licenses/by/ $4.0 /)$.
Featured Application: An elementary method for MMW detection by GDD-LTF combination as single pixel for focal plane arrays.

\begin{abstract}
Performance enhancement of a very inexpensive millimeter-wave (MMW)/terahertz (THz) sensor for MMW/THz imaging systems is experimentally demonstrated in this study. The MMW sensor is composed of a glow discharge detector (GDD) and a light-to-frequency (LTF) converter combination. The experimental results given in this study show an improvement in the performance parameters of the detector element, such as the minimum detectable signal, as well as the signal to noise ratio (SNR) and the noise equivalent power (NEP), when a NIR long-pass filter was inserted between the GDD and the LTF combination. A detailed derivation of the NEP of this unique sensor is presented in this work. Based on this derivation and the experimental measurements, the NEP value was calculated.
\end{abstract}

Keywords: millimeter wave imaging; terahertz sensor; glow discharge detector; light-to-frequency converter; focal plane array; quasi optical design; millimeter wave to visible up-conversion imaging

\section{Introduction}

The lack of rigid, inexpensive, and easy-to-use room temperature MMW/THz sensors and pixels for focal plane arrays (FPAs) makes it difficult to develop some applications, especially those that require $\mathrm{MMW} / \mathrm{THz}$ imaging systems. Currently, one of the main interests of MMW technology is the development of low-cost, fast, highly sensitive, compact, room-temperature sensors. This will allow applications in medicine, communications, homeland security, material science, and space technology [1,2]. The common MMW/THz detectors are semiconductor MMW detectors, Schottky diodes, Golay cells, pyroelectric detectors, bolometers, and microbolometers [3,4]. Semiconductor MMW detectors have high sensitivity and can operate at room temperature [1]. However, they are extremely sensitive to electrostatic discharge (ESD), very expensive and they will be affected and get damaged during instances of incidence by high MMW power. For example, the zero-biased detector (ZBD) WR10ZBD of Virginia Diodes Inc. has a damage input power of $1 \mathrm{~mW}$ [5]. Schottky diodes have a fast response time and lower noise-equivalent power (NEP). However, they are not used for high-power THz pulses, as they are less sensitive at higher frequencies of the $\mathrm{MMW} / \mathrm{THz}$ band, and require much more local oscillator power [6]. Previous studies had proved that weakly ionized plasma (WIP) in a glow discharge neon lamp can be more sensitive to $\mathrm{MMW} / \mathrm{THz}$ radiations and has a shorter response time when compared to that of a Schottky diode [7]. In addition, GDDs can be used to detect 
higher power MMW radiation with both higher and faster response [8]. Hence, we used glow discharge detectors (GDD) as MMW/THz detectors. This will also help to reduce the complexity and cost of the detection system. The cost of individual MMW detectors can play a key role in MMW focal plane array imaging with large numbers of detectors. Fortunately, these GDD devices can run for only about 50 cents each. The GDD is orders of magnitude less expensive- 50 cents compared to the price of other MMW detectors, which is at least hundreds of dollars.

The glow discharge detector (GDD) was found to be one of the typical MMW/THz radiation detectors [3,4,6-12] and pixels in focal plane arrays [13-18] but is orders of magnitude less expensive compared to other known $\mathrm{MMW} / \mathrm{THz}$ sensors [1]. This is particularly important for detector arrays as in imaging. Two detection methods of the GDD were investigated: the first is the electrical current method [3,4,6-11], in which the changes in the bias current of the GDD due to incident MMW/THz radiation were measured. The second is the optical up-conversion method [12,18], in which the changes in the emitted light of the GDD due to the incident $\mathrm{MMW} / \mathrm{THz}$ radiation were measured using a standard optical sensor.

The GDD is a common plasma indicator lamp which can be used as an MMW/THz detector when connected to DC voltage and operating in the abnormal glow regime of the current-voltage (I-V) characteristics as can be seen in Figure 1 [19].

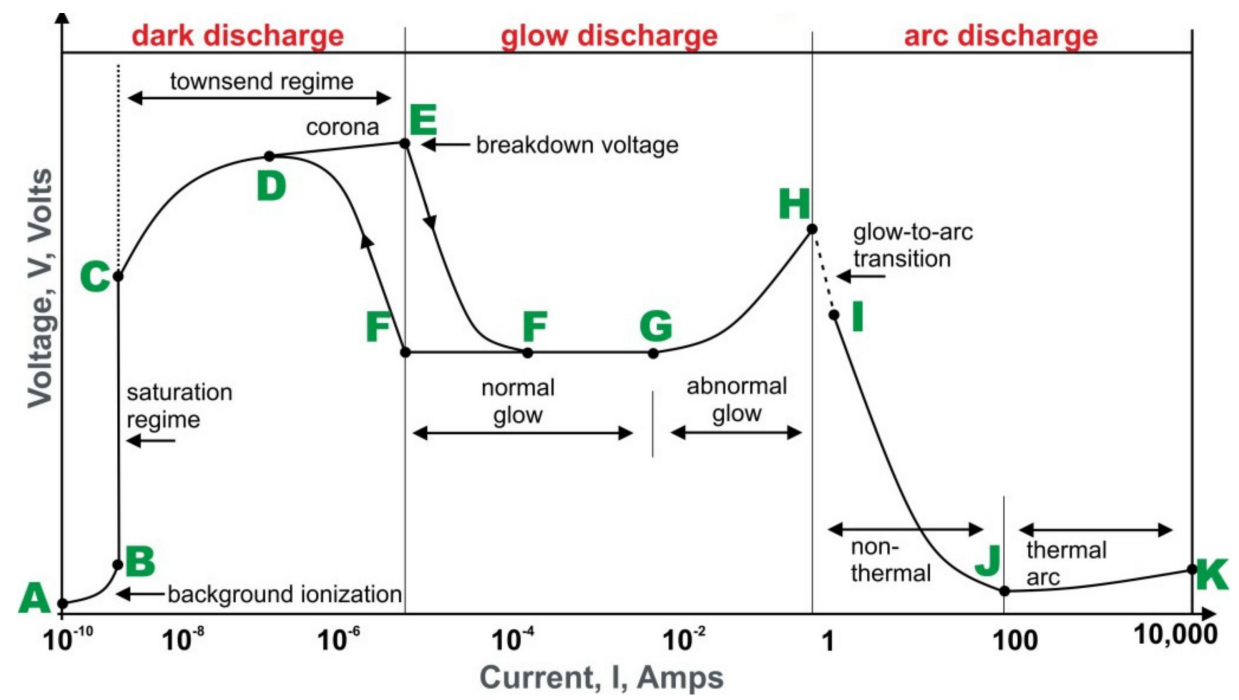

Figure 1. Current-voltage (I-V) characteristics of direct current (DC) electrical discharges.

Breakdown of the gas occurs at voltage $V_{E}$ after the Townsend regime. The current in the plasma increases, entering the normal glow domain (point $E$ to point $F$ on the curve of Figure 1). Further increase of the current will bring us to the abnormal glow regime (point $\mathrm{G}$ to $\mathrm{H}$ on the curve of Figure 1) where the detection of $\mathrm{MMW} / \mathrm{THz}$ radiation is taking place. The GDD's detection mechanism is based on a slight change in the bias current between the two electrodes of the lamp due to the electric field of the MMW/THz electromagnetic radiation being incident on the GDD. The expression for the change in bias current of the GDD due to incident MMW/THz radiation is given by [20]:

$$
\Delta I(t)=\frac{G \cdot q^{2} \cdot V \cdot n}{V_{i} \cdot m} \cdot\left(\frac{\tau}{\tau_{i}}\right) \cdot \eta_{0} \cdot P_{D} \cdot\left(\frac{V}{V^{2}+w^{2}}\right) \cdot\left(1-e^{\frac{-t}{\tau}}\right)
$$

where $G \approx \frac{\exp \left(2 \cdot v_{i} \cdot t_{d}\right)}{2 \cdot v_{i} \cdot t_{d}}$ is designated as internal signal amplification (calculations of $G$ indicate that it can be on the order of a million), $q$ is the electron charge, $V$ is average electron velocity, $n$ is the electron density, $V_{i}$ is gas ionization potential, $m$ is the electron mass, $\tau$ is time response to create current changes, $\tau_{\mathrm{i}}$ is the time between ionization collisions of electrons with gas atoms, $\eta_{0}$ is free space wave impedance, $P_{D}$ is the incident $\mathrm{MMW} / \mathrm{THz}$ 
radiation power density on the detector, $v$ is the electron-neutral atom elastic collision frequency, $\omega$ is the electromagnetic radiation frequency, $t_{d}$ is the average electron drift time to the anode, and $v_{i}$ is ionization collision frequency. The dominant $\mathrm{MMW} / \mathrm{THz}$ detection mechanism inside the plasma was found to be enhanced cascade ionization rather than diffusion current $[4,20-22]$. The enhanced cascade ionization mechanism increases the current while the diffusion current mechanism decreases it. The response time of the plasma inside the GDD is on the order of picoseconds. However, the electronic response time is limited by GDD's electrode parasitic impedance and the electronic circuit to about $1 \mu \mathrm{s}[16]$.

According to the glow discharge theory, the light emission intensity is directly linked to the detection of the MMW/THz radiation. GDD illumination is originated from the recombination between free electrons and positive ions which occur mostly around the negative glow area [20], which is adjacent to the cathode. This area is where the DC electric field and the total electron energy are at a maximum. Furthermore, when the frequency of the MMW radiation is equal to the inelastic collision rate, maximum response of the GDD is obtained (see Equation (1)) [20]. The radiation affects mostly the area around the cathode dark space, which is adjacent to the cathode.

A new detection mechanism for MMW/THz radiation detection using GDD, named up-conversion method, was suggested [17]. The up-conversion method (optical detection) refers to the detection of changes in the emitted light from the GDD due to incident $\mathrm{MMW} / \mathrm{THz}$ radiation, rather than the changes in its electrical current (electronic detection). In the up-conversion method, a basic photodiode or any basic light sensor is required in order to measure the changes in the GDD emitted light. The advantages of the upconversion method are: (1) a very fast response time which is limited by the light sensor response, (2) a lower internal noise compared to the electric detection [17], and (3) it is very inexpensive.

The spectral emission of typical neon lamp and its energy levels are shown in Figure 2 [23].

Figure 2a shows a spectral measurement of a typical neon lamp carried out by ISF $125 \mathrm{M}$, Bruker Inc. The measurement accuracy of the radiated lines is better than $1 \mathrm{GHz}$ [23]. The radiated energy levels of Figure $2 b$ are in excellent agreement with the experimental results of Figure 2a.

In preliminary research aiming to investigate the influence of MMW radiation on the GDD emitted light spectrum, it was discovered that the strongest influence of the $\mathrm{MMW} / \mathrm{THz}$ on the GDD emitted light spectrum is located in the NIR around $800 \mathrm{~nm}-$ $1000 \mathrm{~nm}$ (see solid blue line in Figure 3). Furthermore, almost no influence of the MMW/THz around $500 \mathrm{~nm}-600 \mathrm{~nm}$ was noticeable. Figure 3 shows preliminary experimental spectrum measurements and analysis of the emitted light from GDD N527 (of International Light Inc.), without MMW radiation (orange solid line) and with MMW radiation (blue solid line). Despite the many advantages mentioned above regarding the GDD and LTF combination pixel as a useful and robust MMW detector, further improvement to the detector pixel sensitivity and to the SNR are required. This paper proposes the use of an optical long-pass filter as a part of the GDD and LTF pixel component, thus enhancing the pixel detector sensitivity as well as its signal-to-noise ratio and thus improving performance of the suggested THz/MMW radiation sensor or pixel. The addition of the long-pass filter is shown here to improve the total SNR of the detected MMW signal. This improvement is due to two reasons. The first reason is the improvement in the noise level due to the filtering of the bias illumination noise of the GDD and the second reason is the increase in the signal level due to back reflection from the filter body towards the GDD cross-section. 


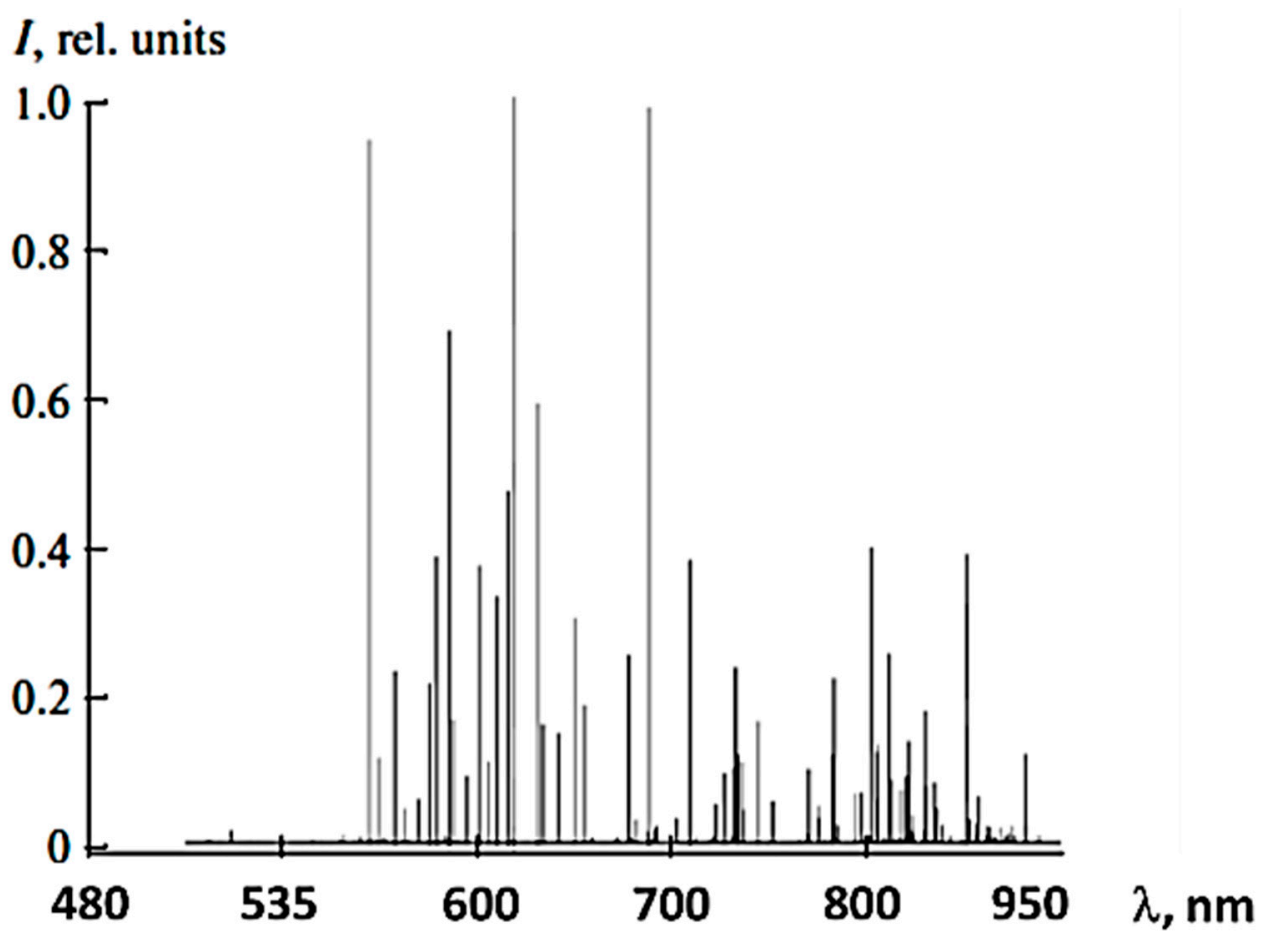

(a)

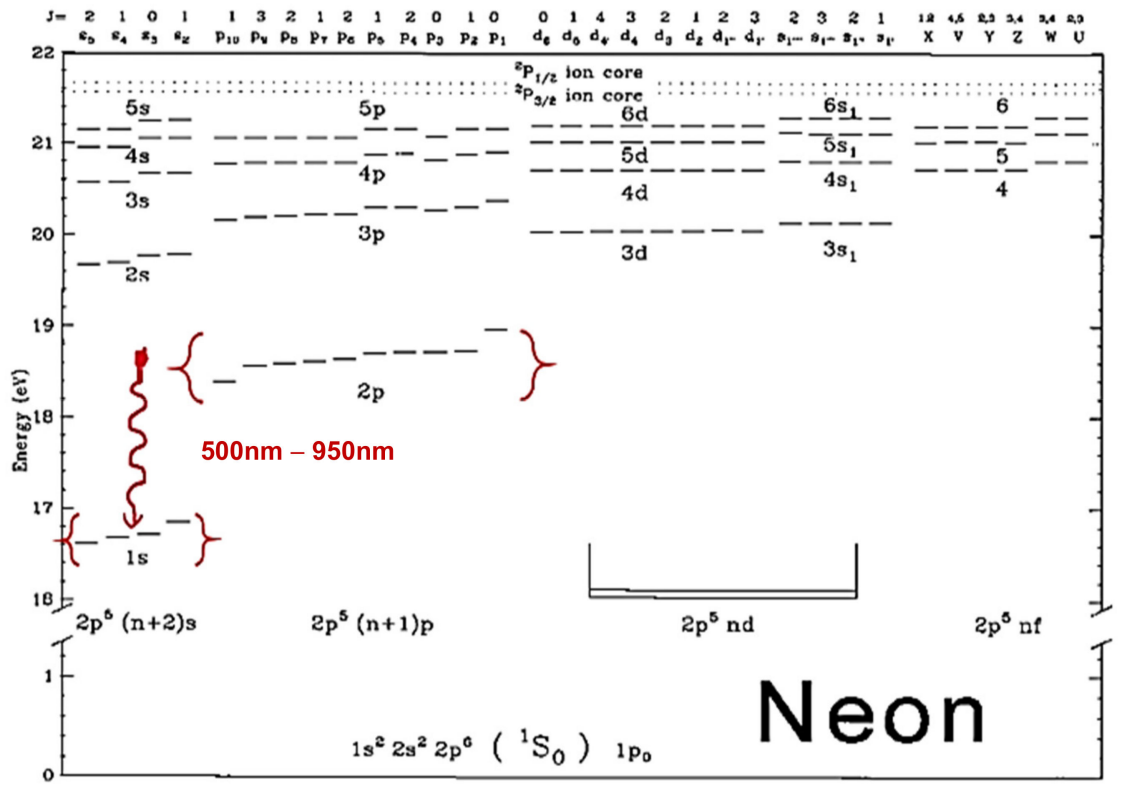

(b)

Figure 2. Typical measured spectral emission of neon lamp (a) and energy levels of neon. The radiated transitions are marked in red $(\mathbf{b})[23,24]$. 


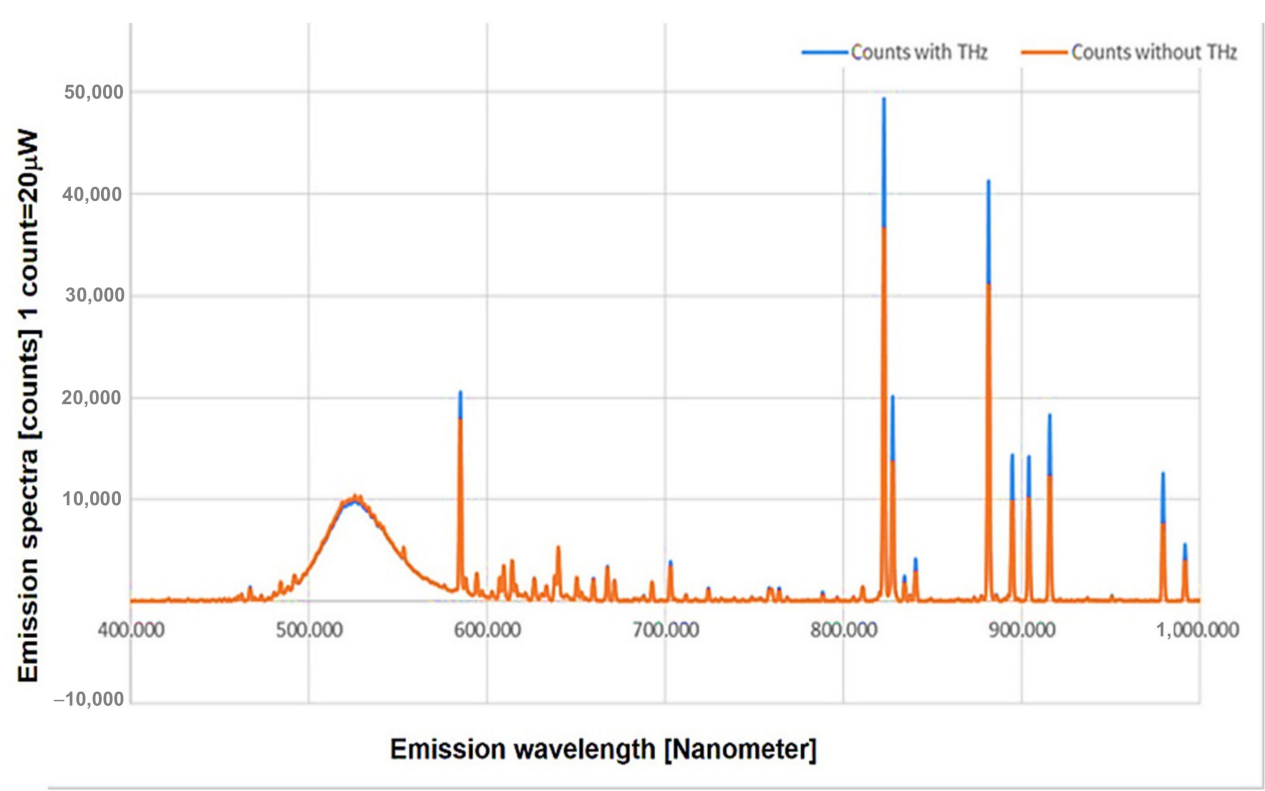

Figure 3. Emission spectra of GDD N527 with $\mathrm{MMW} / \mathrm{THz}$ influence (blue line) and without $\mathrm{MMW} / \mathrm{THz}$ influence (orange line). One count is equal to $20 \mu \mathrm{W}$. (Courtesy of Prof. Dr. Asaf Behzat Sahin, Electrical and Electronics Engineering Department, Ankara Yildirim Beyazit University, Turkey).

\section{Noise and Noise Equivalent Power Calculations}

In up-conversion detection (or optical detection), the electric field of the $\mathrm{MMW} / \mathrm{THz}$ radiation causes a slight change in the electrical bias current, which increases the emitted light from the GDD [17]. This increase in the GDD light intensity derives from de-excitation or recombination rather than electron excitation. The DC bias field accelerates positive ions towards the cathode, which produces a secondary electron emission from the cathode. The large densities of these secondary electrons and positive ions yield a large increase in recombination near the cathode, which generates an increase in GDD light intensity at the negative glow region [20,22]. In this sensor system, the input signal to the GDD is an MMW/THz wave, and the output signal from the GDD is an optical wave instead of an electronic current. This upconverted wave was shown to have a much faster response time [19]. Those emitted light changes in the GDD intensity are detected by the LTF component. The output signal of the LTF is an electronic square wave frequency which is proportional to the GDD emitted light changes. The electronic response time then depends on the speed of the opto-electronic components. In the present case, that component is the LTF converter.

The noise mechanism of the suggested sensor evolves both from the GDD and from the LTF. The GDD is a plasma device, in which the total noise power evolves from two noise sources. The first is Johnson-Nyquist noise or thermal noise given by:

$$
N_{0}=k T_{e}
$$

where $N_{0}$ is the noise spectral density $[\mathrm{W} / \mathrm{Hz}], k$ is the Boltzmann constant $[1.380649 \times 10-23 \mathrm{~J} / \mathrm{K}]$, and $T_{e}$ is the electron temperature $[\mathrm{K}]$. The second is shot noise power caused by random fluctuations of the electric DC current in the plasma given by:

$$
S=2 e I_{D C}
$$

where $S$ is the shot noise spectral density, $e$ is the electron charge $[C]$, and $I_{D C}$ is the electric current in the plasma. Usually, the dominant noise source in the plasma is the thermal noise due to high $T_{e}$, which is on the order of $T_{e} \cong 40,000 \mathrm{~K}$. 
While the electronic detection (or electrical detection) is controlled by ionizing collisions and by electron energy or temperature, the up-conversion detection is controlled by positive ion-secondary electron recombination processes and is affected primarily by positive ion energy or temperature. Due to the larger mass of the ion as compared to the electron mass, the positive ion temperature is orders of magnitude less than the electron temperature. Thus, up-conversion detection Johnson noise is significantly less than the electronic detection Johnson noise [14]. Furthermore, according to the literature, the electron temperature in plasma, $T_{e}$, is on the order of $30,000-40,000 \mathrm{~K}$, while the ion temperature $T_{i}$ is around $500 \mathrm{~K}$ [25]. Therefore, the noise generated in up-conversion detection is composed of both thermal noise (using $T_{i}$ ) and shot noise (due to recombination between secondary electrons and positive ions near the GDD cathode). The influence of those noise sources on the GDD emitted light fluctuations can be measured with a basic optical power meter. For example, measuring the irradiance of the GDD in two successive measurements and subtracting them, yields the total noise in the GDD irradiance. The GDD irradiance is the input signal to the LTF component which is attached to it. Thus, the output frequency noise, $\Delta f_{n}$, is the subtraction of two successive output frequency measurements. Furthermore, the influence of the $\mathrm{MMW} / \mathrm{THz}$ radiation, $\Delta f_{M M W}$, on the output frequency can be measured the same way if we subtract two successive measurements where the first one is with MMW radiation and the second one is without MMW radiation.

The LTF converts input optical irradiance, $E_{e}$, to the electric TTL levels frequency, $f_{0}$. $R_{e}$ is the irradiance responsivity. The dark frequency of the LTF is $f_{D}$ is $2 \mathrm{~Hz}$. The NEP of the suggested sensor can be calculated according to:

$$
N E P=\frac{\left(\Delta f_{n}+f_{D}\right)}{\operatorname{Re} \sqrt{B}}
$$

where $B=1 \mathrm{MHz}$ is bandwidth of the LTF [26]. Note that the GDD modulation bandwidth is very high, on the order of several GHz [12,18], attesting to the GDD speed of response. In these speed measurements, speed was limited by the optoelectronic detector and not by the GDD plasma.

\section{Experimental Set Up and Components}

The experimental set up is composed of an MMW source manufactured by Virginia Diodes Inc (VDI TX272), a quasi-optic design based on one off-axis parabolic mirror (OPM), a convex lens, a timer/counter type NI USB-6341 manufactured by national instruments (NI), a glow discharge detector N527 (of International Light Inc.), a Light-to-frequency converter (LTF) component TSL237 (of ams AG), and a near-infrared (NIR) Long-Pass Filter (LPF) with cutoff wavelength of $550 \mathrm{~nm}$ with an optical density 6 . The experimental set up is shown in Figure 4. Careful inspection of the spectral analysis of the GDD irradiance (see Figure 3), reveals that the influence of MMW / THz radiation is noticeable in the spectral range of $800 \mathrm{~nm}$ to $1000 \mathrm{~nm}$. Furthermore, no significant influence was noticeable at lower wavelengths [27]. Thus, a $550 \mathrm{~nm}$ long-pass filter was selected to cut out most of the background irradiance of the GDD that doesn't contribute to the detection of $\mathrm{MMW} / \mathrm{THz}$ radiation.

The filter is mounted in an engraved metal ring that increases the thickness of the filter. The long-pass filter (FEL0550, of Thorlabs) was placed between the GDD and the LTF as can be seen in Figure 4. The GDD neon lamp used in this set-up is N527 manufactured by International Light Technologies. The neon lamp type N527 is shown here to be very sensitive to MMW detection using the up-conversion method. The LTF converts the emitted light of the GDD to TTL levels of square wave frequency, where the square wave frequency increases proportionately with the GDD light intensity. A simple and inexpensive LTF component, TSL237 (of ams AG) was used to convert the GDD light to frequency. The TSL237 has the following parameters: 2.7-5.5 V operating voltage, $320 \mathrm{~nm}$ to $1050 \mathrm{~nm}$ spectral response range, and output square wave frequency dynamic range of $1 \mathrm{~Hz}$ to $1 \mathrm{MHz}$. The GDD was placed with its head toward the incident MMW radiation (see 
Figure 4). Therefore, its effective detection cross-section is a circle with a diameter of about $1 \mathrm{~mm}$. Propagation calculations based on the Gaussian beam approximation, show that about $0.1 \%$ of the transmitted power is received by the GDD effective cross-section [28] Thus, the maximum MMW radiation power at the cross-section of the GDD is about $3.42 \mathrm{~mW}$. A timer/counter type NI USB-6341 was used to measure the output frequency of the LTF as can be seen in Figure 4. The timer/counter was controlled and operated by a LABVIEW code. The MMW source is located at the focal point of the OPM. The MMW radiation carrier frequency is $100 \mathrm{GHz}$. This carrier wave was modulated using $\mathrm{ON} / \mathrm{OFF}$ keying $(\mathrm{OOK})$ at a frequency of $2 \mathrm{~Hz}(1 \mathrm{~s}$ with the radiation in the $\mathrm{ON}$ state and $1 \mathrm{~s}$ with the radiation in the OFF state). The maximum output power of the MMW source is $600 \mathrm{~mW}$. The output power of the MMW source can be varied by using the user-controlled

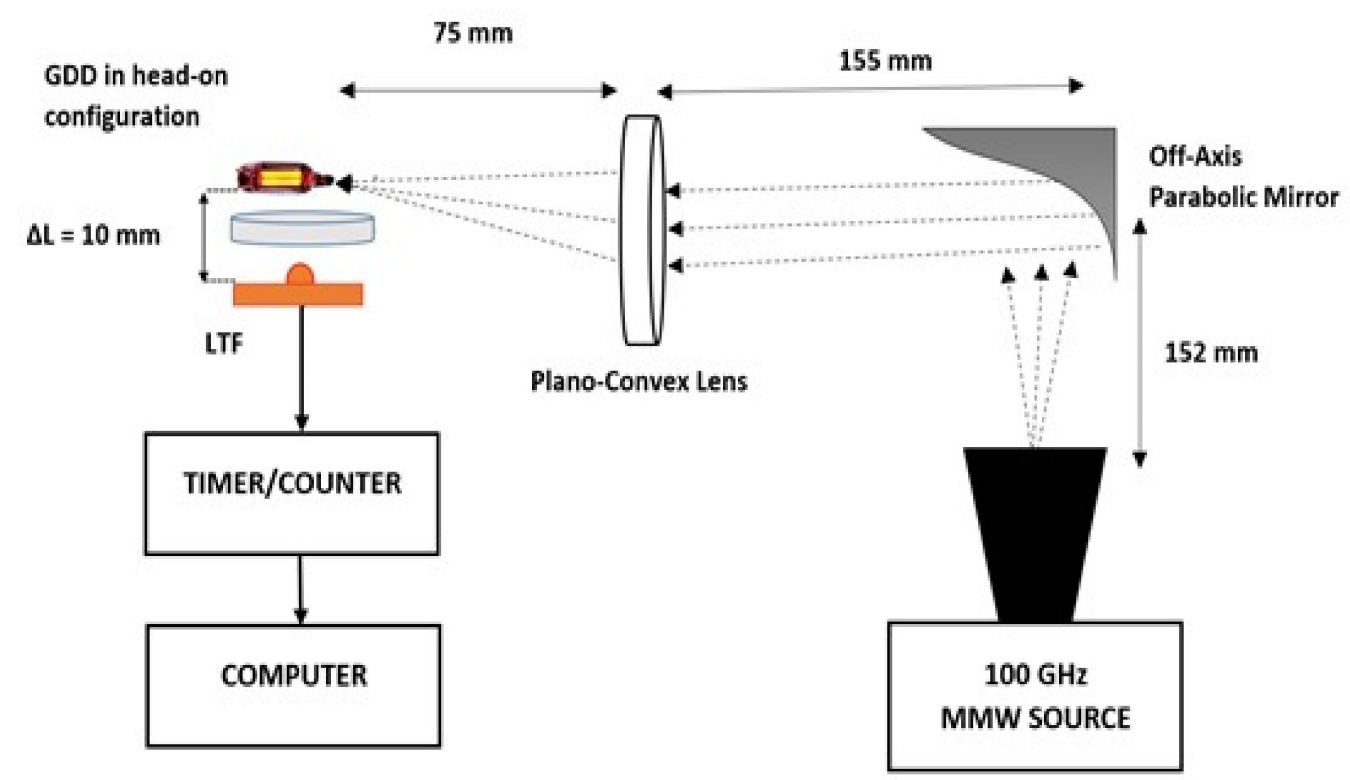

Figure 4. Experimental set up used for the minimum detectable signal and responsivity measurements.

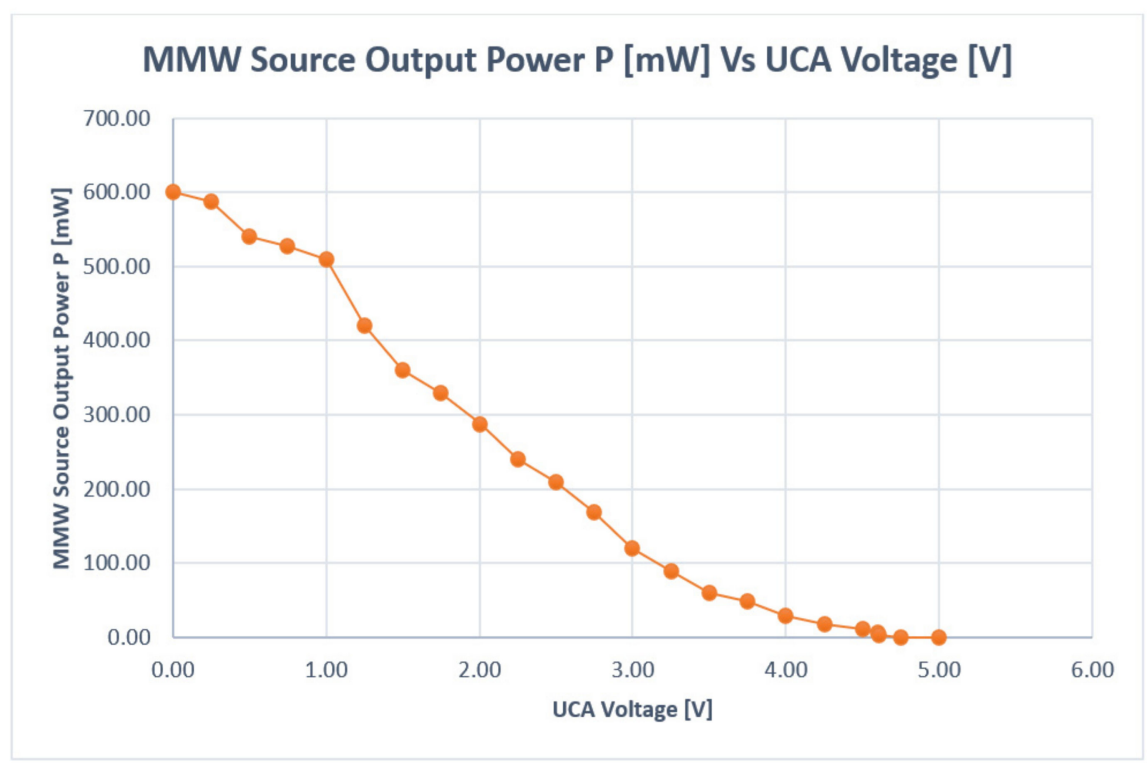

Figure 5. Variation in MMW source power according to the UCA input.

Initially, the voltage across the GDD was measured through electrical detection by varying the user-controlled attenuation (UCA) input of the MMW source from $0 \mathrm{~V}$ to $5 \mathrm{~V}$ so as to ensure maximum radiation will incident on the GDD cross-section. A horn antenna is 
used to couple out the MMW radiation to free space. The electrical detection characteristics of the GDD when exposed to MMW radiation are shown in Figure 6.

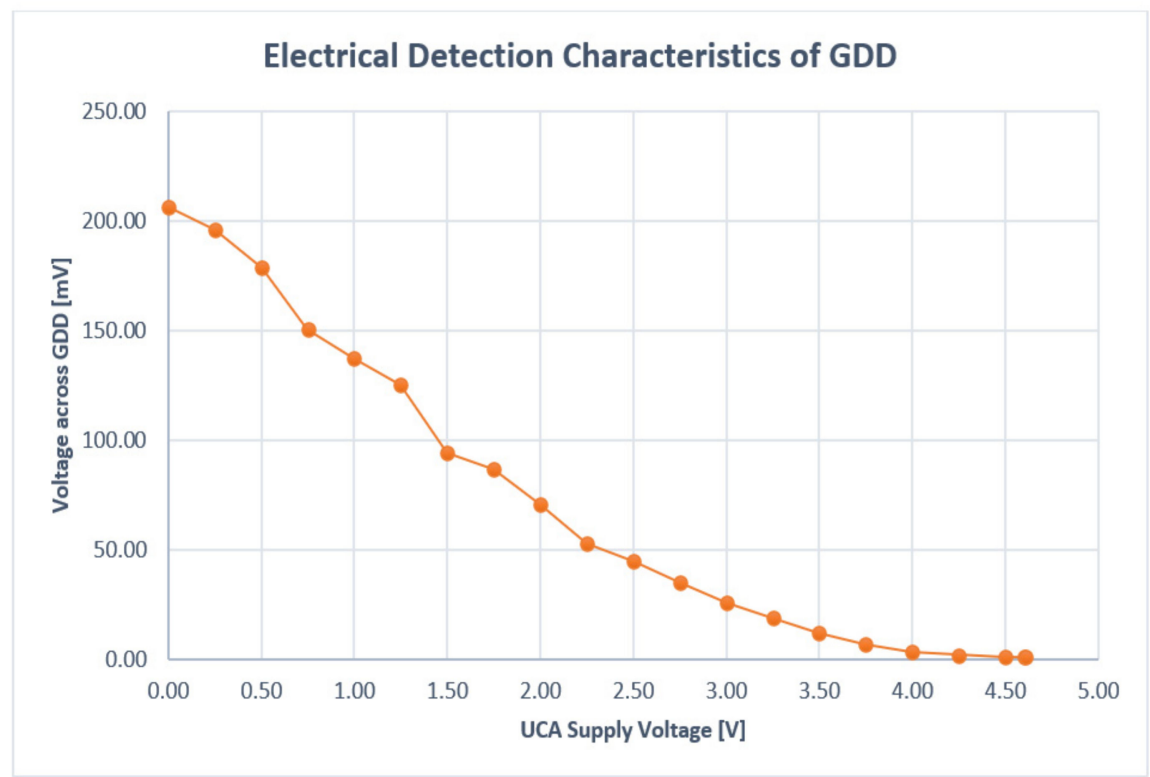

Figure 6. Voltage across GDD as a function of UCA voltage.

The difference in LTF output with respect to the variation in the GDD bias current $I_{D C}$ is also measured as shown in Figure 7.

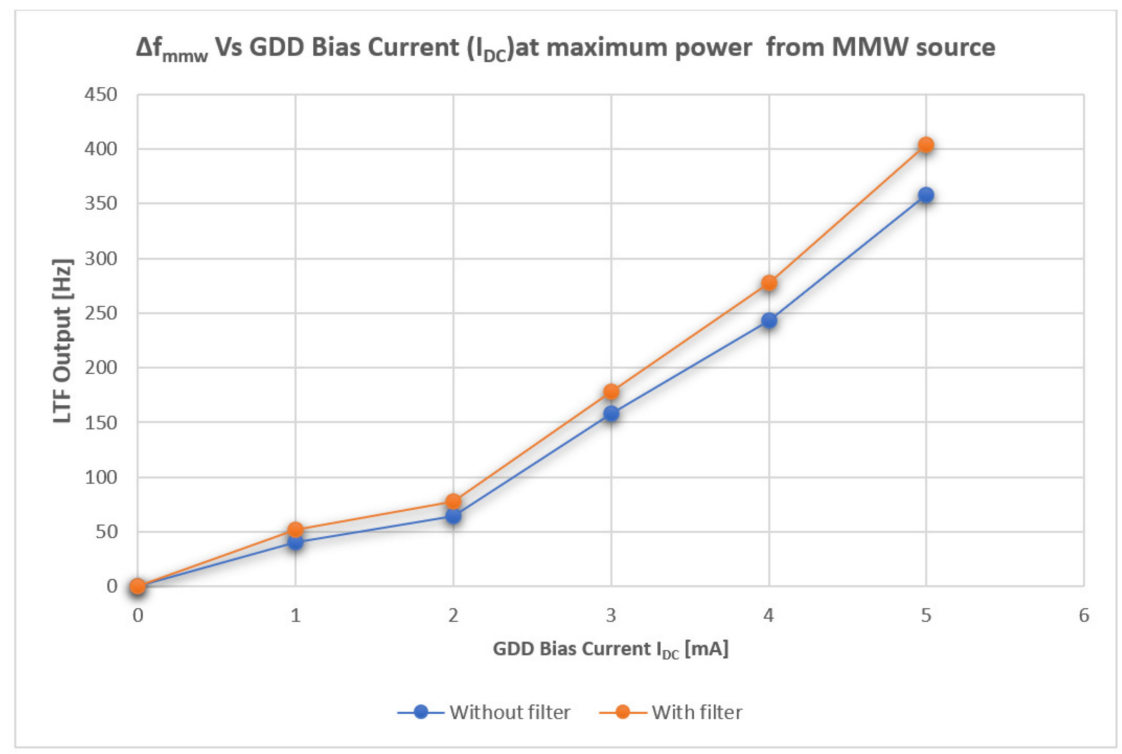

Figure 7. LTF output $\Delta \mathrm{f}_{\mathrm{mmw}}$ as a function of GDD bias current $\left(\mathrm{I}_{\mathrm{DC}}\right)$.

A collimated beam is obtained after the first OPM and it propagates towards the aspheric lens. The GDD and the LTF combination are located at the focal point of the second OPM. The output frequency of the LTF is measured using a computerized system as described above.

\section{Experimental Results}

\subsection{Noise Measurements}

To perform noise measurements, we used the set-up shown in Figure 8. By measuring the deviation in the LTF frequency as a result of the GDD noise, with and without the long 
pass filter, we were able to evaluate the improvement in the minimum detectable MMW signal using the long-pass filter. The measurement conditions used in this experiment were: DC bias current of $5 \mathrm{~mA}$, and a distance between the LTF and GDD $(\Delta L)$ of $10 \mathrm{~mm}$. The experimental results in Figure 9 show the influence of the $550 \mathrm{~nm}$ long-pass filter on the frequency deviation of the LTF. As expected, by deleting GDD bias light below $550 \mathrm{~nm}$, the noise is noticeably reduced.

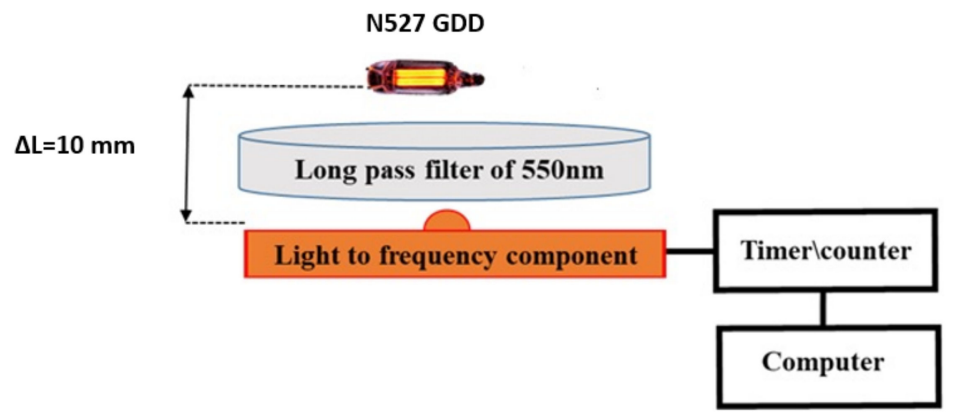

Figure 8. Experiment setup of the noise measurements.

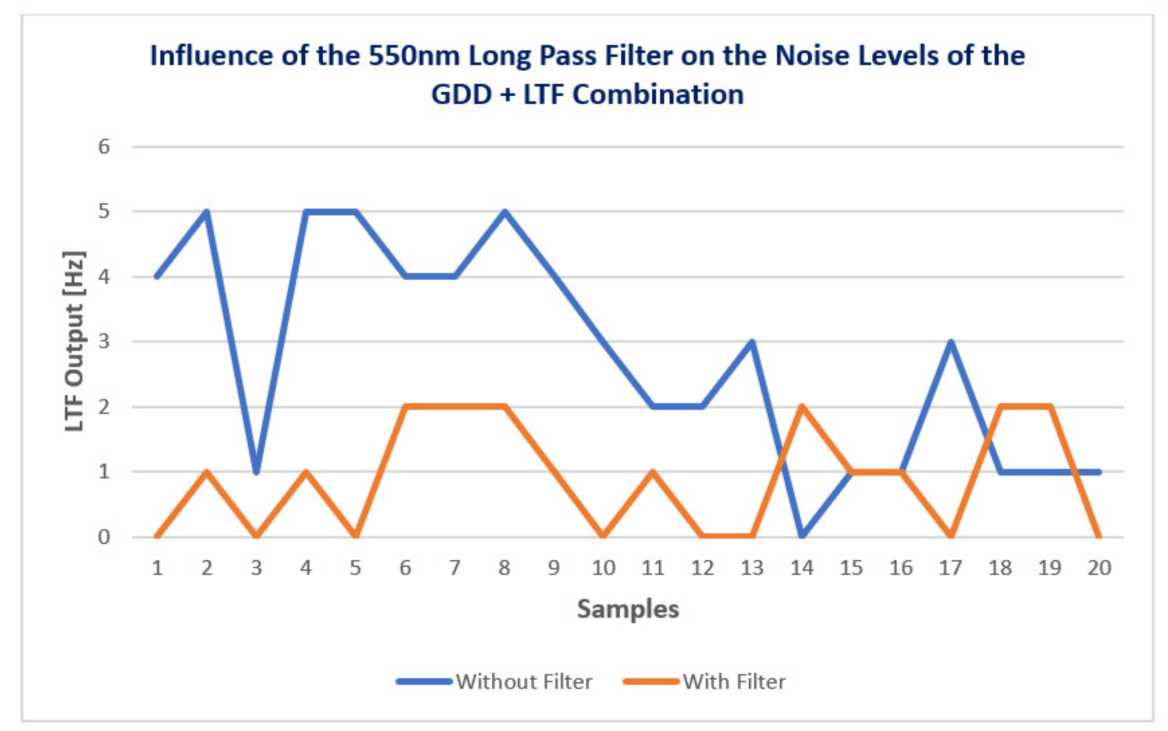

Figure 9. The influence of the $550 \mathrm{~nm}$ long pass filter on the noise levels of the GDD + LTF pixel.

\subsection{Minimum Detectable MMW Signal Source Measurements}

The minimum detectable MMW signal was measured by attenuating the output power of the MMW source until the point where there was no effect of the MMW power on the difference in the output frequency generated from the LTF. The minimum detectable MMW power incident on the N527 GDD's effective cross-section was measured for $T_{\text {Gate }}=100 \mathrm{~ms}$, where $T_{\text {Gate }}$ is defined as the LTF counting time for the case where the MMW radiation is in the ON state and also in the OFF state. The results of the minimum detectable signal (MDS) for these cases are presented in Table 1.

Table 1. Minimum detectable MMW source signal (MDS) measurement results with and without $550 \mathrm{~nm}$ long pass filter at $T_{\text {gate }}=100 \mathrm{~ms}$.

\begin{tabular}{ccc}
\hline & with $550 \mathbf{~ n m}$ Filter & without Filter \\
\hline$M D S$ at $\left(T_{\text {gate }}=100 \mathrm{~ms}\right)$ & $0.02 \mathrm{~mW}$ & $0.1 \mathrm{~mW}$ \\
\hline
\end{tabular}

The deviation in the output frequency was measured in a dark room such that the light incident on the LTF was only the light incident from the GDD. Twenty LTF output 
frequency measurements were taken using a single pixel in a consecutive manner of one with MMW radiation and one without it. Every sample was taken in $T_{\text {gate }}=100 \mathrm{~ms}$. The subtraction between two consecutive samples gives the MMW influence on the output frequency. The measurement results are summarized in Table 1 for GDD N527.

The effect of the $550 \mathrm{~nm}$ long-pass filter on the GDD was measured, with the setup shown in Figure 4, at full MMW source output power of $600 \mathrm{~mW}$, transmitted toward the GDD and LTF pixel. Results are shown in Figure 10. In this measurement, $T_{\text {gate }}$ was chosen to be $1 \mathrm{sec}$. The filter actually increases the LTF square wave frequency, thus increasing SNR beyond the improvement generated by the reduction in noise.

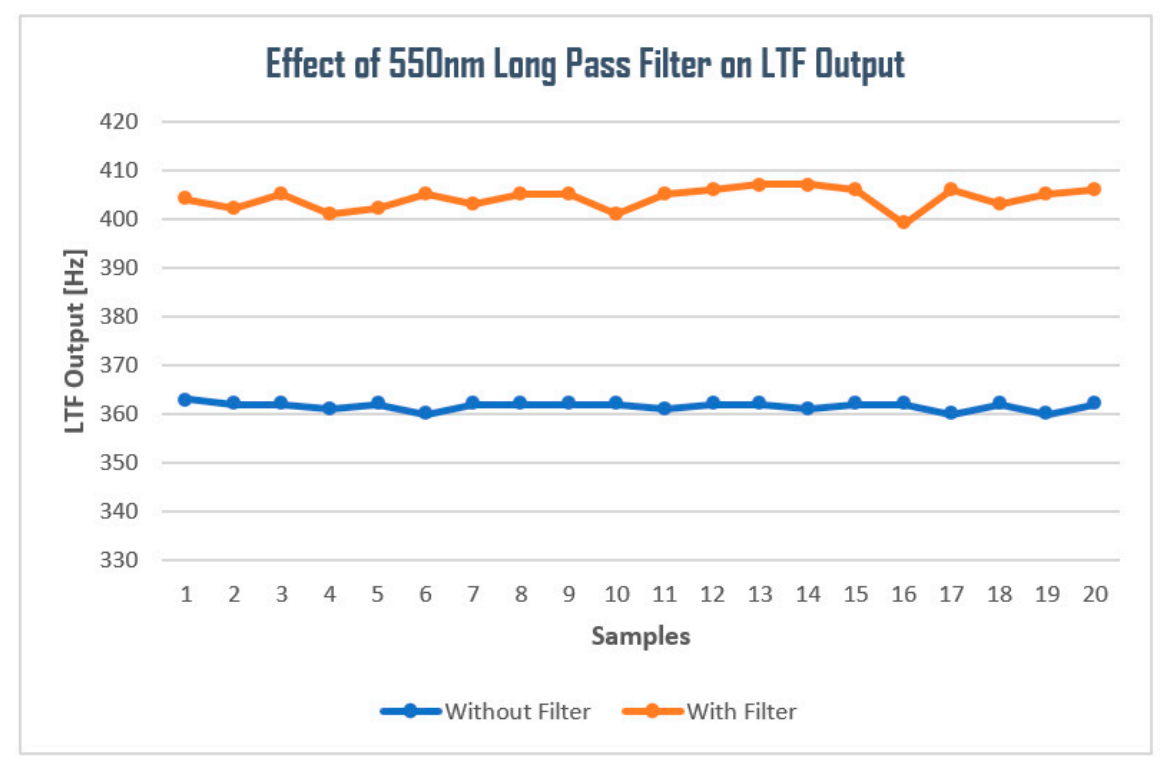

Figure 10. The effect of the $550 \mathrm{~nm}$ long pass filter on the GDD detector at full MMW source output power.

\subsection{Calculation of MMW Power Incident on GDD Cross Section}

The initial alignment of the quasi-optical setup was carried out using a laser source that was placed instead of the MMW source. Further alignments were performed by adjusting the position of quasi-optical dishes and the GDD in order to receive a stronger MMW signal. The MMW source horn antenna was located at the focal point of the OPM. The Gaussian beam approximation was used to calculate the beam waist (Equation (5)), and the total power collected by the GDD cross-section is calculated in Equation (6).

$$
\omega(z)=\omega_{0} \sqrt{1+\left(\frac{z}{z_{R}}\right)}
$$

where $z_{R}=\frac{\pi \omega_{0}}{\lambda}$

$$
P=P_{0}\left(1-e^{-\frac{D^{2}}{2 \omega(z)^{2}}}\right)
$$

where $\omega(z)$ is beam waist, $z$ is the distance of propagation, $\lambda$ is the MMW wavelength, $Z_{R}$ is the Rayleigh range of a Gaussian beam, $P_{0}$ is the total beam power emitted from the MMW source, and $D$ is the diameter of the GDD.

According to VDI's nominal horn specifications, the MMW beam waist radius refers to the input of the conical horn antenna and is fixed by that geometry to $\omega_{0}=6.2 \mathrm{~mm}$. The MMW source was placed in the focal point of the OPM and the GDD was placed at the focal point of the convex lens. The approximate beam waist can be calculated for a given position in the quasi-optical setup, using the transformation of the complex beam parameter $\mathrm{q}_{1}$ at the output of the MMW source $(W=6.2 \mathrm{~mm}$ and $R=\infty)$ by the ABCD 
transfer matrix for paraxial rays. The complex beam parameter $q_{2}$ can then be calculated at that given position using Equation (4). Knowing the complex beam parameter $q_{2}$ can allow the calculation of the beam waist $(W)$ and the beam radius of curvature $(R)$ at that given position using Equation (5).

$$
\begin{gathered}
{\left[\begin{array}{ll}
A & B \\
C & D
\end{array}\right]=\left[\begin{array}{cc}
1 & d_{3} \\
0 & 1
\end{array}\right]\left[\begin{array}{cc}
1 & 0 \\
-1 / F_{\text {Lens }} & 1
\end{array}\right]\left[\begin{array}{cc}
1 & d_{2} \\
0 & 1
\end{array}\right]\left[\begin{array}{cc}
1 & 0 \\
-1 / F_{\mathrm{OPM}} & 1
\end{array}\right]\left[\begin{array}{cc}
1 & d_{1} \\
0 & 1
\end{array}\right]} \\
q_{2}=\frac{A q_{1}+B}{C q_{1}+D} \\
\frac{1}{q_{2}}=\frac{1}{R}-\frac{i \lambda}{\pi W^{2}}
\end{gathered}
$$

The parameters in Equation (7) are as follows:

$d_{1}$ is the distance between the transmitter and the OPM $(152 \mathrm{~mm}), F_{\text {Lens }}$ is the focal length of the lens $(75 \mathrm{~mm}), F O P M$ is the focal length of both of the OPM components $(152 \mathrm{~mm}), d_{2}$ is the distance between the OPM and lens $(155 \mathrm{~mm})$, and $d^{3}$ is the distance between the lens and the GDD $(75 \mathrm{~mm})$.

\subsection{Calculation of the Irradiance Responsivity}

The calculation of the irradiance responsivity $R e$ was carried out using the quasioptical setup shown in Figure 4. Apart from the long pass filter, in our responsivity measurement, we need to characterize only the relationship between the GDD and the LTF without any medium between them. The irradiance responsivity measurement was performed by modifying the MMW source output power from 0-100 percent in leaps of $5 \%$. To do that we used the user-controlled attenuation (UCA) input of the MMW source in order to control the source attenuation by providing different voltages in its input where $0 \mathrm{~V}$ is zero attenuation and $5 \mathrm{~V}$ is max attenuation. To improve accuracy, 40 samples of $f_{m m w}$ (output frequency of LTF when exposed to MMW radiation) were performed, then a subtraction was performed to each pair of consecutive samples, one with the MMW source in ON mode and one with it in OFF mode, which yielded $20 \Delta f_{m m w}$ (change in consecutive samples of output frequency of LTF when exposed to MMW radiation) samples that were averaged to produce a single sample of $\Delta f_{m m w}$ as shown in Figure 11.

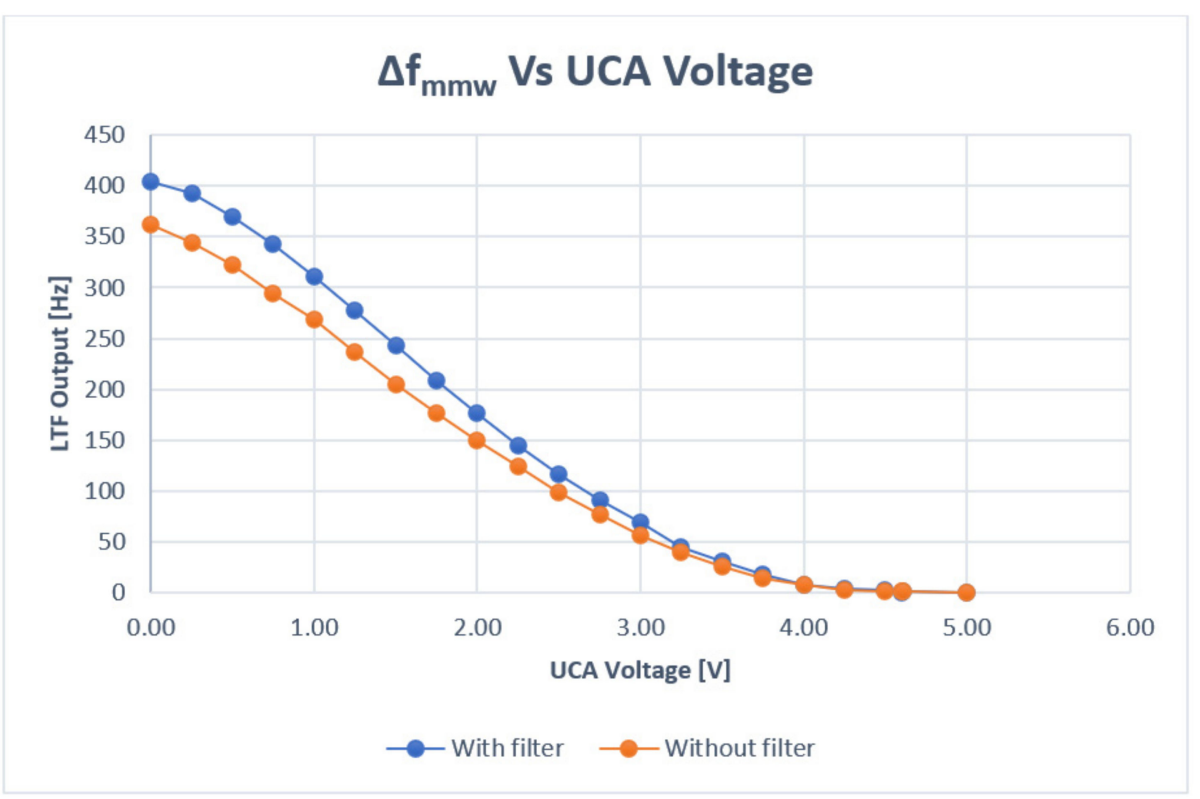

Figure 11. Variation in the output frequency of LTF in accordance to the UCA input. 
Each sample was taken in $T_{\text {gate }}=100 \mathrm{~ms}$. The results from the responsivity measurement are shown in Figure 12, where $P_{G D D}$ is the amount of MMW radiation power hitting the cross-section of the GDD.

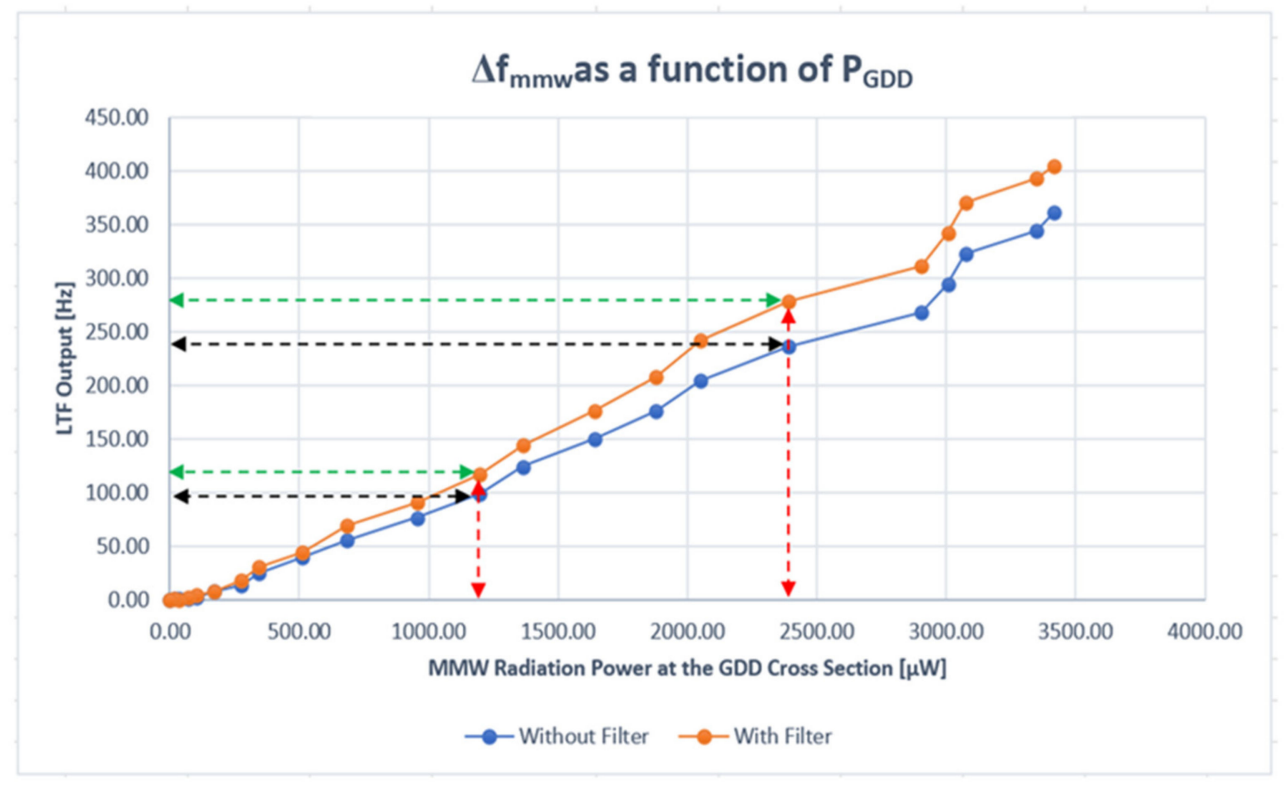

Figure 12. Difference in output frequency $\Delta f_{m m w}$ as a function of MMW radiation power hitting the cross section of the GDD. below:

The irradiance responsivity is given by calculating the slope of the graph as given

(a) With filter

$$
R e=\frac{(278-116.9)}{(2390.8-1195.4) \times 10^{-6}}=134.77 \mathrm{KHz} / \mathrm{W}
$$

Since only $0.1 \%$ of the MMW source power reaches the GDD, the irradiance responsivity is $\operatorname{Re} \times \frac{1}{0.001}$.

Hence $\operatorname{Re}=134.77 \mathrm{MHz} / \mathrm{W}$ (with filter).

(b) Without filter

$$
R e=\frac{(236.9-99.05)}{(2390.8-1195.4) \times 10^{-6}}=115.32 \mathrm{KHz} / \mathrm{W}
$$

Since only $0.1 \%$ of the MMW source power reaches the GDD the irradiance responsivity is $\operatorname{Re} \times \frac{1}{0.001}$.

Hence $R_{e}=115.32 \mathrm{MHz} / \mathrm{W}$ (without filter).

\subsection{Calculation of NEP}

Substituting in Equation (4) the value B = $1 \mathrm{MHz}$ (bandwidth of the LTF), the dark frequency of the LTF is $f_{D}=2 \mathrm{~Hz}$, the average value of the output frequency noise with filter is $\Delta \mathrm{fn}=0.9 \mathrm{~Hz}$, and without filter is $\Delta \mathrm{fn}=2.75 \mathrm{~Hz}$, resulting in the NEPs:

(a) With filter

$$
N E P=\frac{(0.9+2)}{134.77 \times 10^{9}}=2.15 \times 10^{-11} \mathrm{~W} / \sqrt{ } \mathrm{Hz}
$$


(b) Without filter

$$
N E P=\frac{(2.75+2)}{115.32 \times 10^{9}}=4.12 \times 10^{-11} \mathrm{~W} / \sqrt{ } \mathrm{Hz}
$$

\section{Discussion}

The results show great promise for the future of GDDs as MMW detectors. In this proof of concept, we decreased the amount of white bias light noise received by the MMW detector and improved the sensitivity to MMW radiation. These phenomena can be explained by three main factors. First, by removing the DC bias light below $550 \mathrm{~nm}$, the long pass filter lowered the noise floor of the setup that enables us to increase our LTF measurement precision. Second, by adding a filter to the detector set-up, internal reflections from the filter back to the GDD and from the GDD forward to the filter occur which contributes to the increase of signal together with changing the LTF operational point due to the decrease in the background light of the GDD. Third, the decrease in perceived GDD illumination power caused by the filter brings about the decrease of LTF working voltage which increases the accuracy of the pulse rate frequency generated by the LTF semiconductor device. In other words, at low SNR such as when measuring minimum detectable signal, LTF output pulse rate is further reduced because of the low SNR than it should be. At high SNR, the output LTF pulse rate is increased more than expected from the increased GDD light intensity alone. A setup of a GDD and LTF is shown here to be very sensitive to MMW radiation. Following all advantages achieved, we can now create a better and more sensitive GDD and LTF MMW detector in multiple or singular systems.

\section{Conclusions}

The setup of a GDD and LTF is shown here to be very sensitive to MMW radiation. By adding the long-pass filter, the noise level in the output from the GDD is reduced. Thus, a higher value of SNR is obtained. By using the data from Figure 12 and Equation (4), the NEP can be calculated for both incidence with and without long-pass filter, where the average value of the output frequency noise with filter is $\Delta f_{n}=0.9 \mathrm{~Hz}$, and without filter is $\Delta f_{n}=2.75 \mathrm{~Hz}$. NEP is $2.15 \times 10^{-11} \mathrm{~W} / \sqrt{ } \mathrm{Hz}$ (with filter) and $4.12 \times 10^{-11} \mathrm{~W} / \sqrt{ } \mathrm{Hz}$ (without filter). The GDD and LTF setup can be used as a pixel in a focal plane array (FPA), such that the pixel readout can be performed using a digital system.

\section{Future Works}

The GDD-LTF combination with a filter was found to be a good choice for MMW/THz detection as it can reduce NEP, thereby improving the SNR. Because of the frame of the 550 $\mathrm{nm}$ NIR filter, the minimum separation between GDD and LTF used here is $10 \mathrm{~mm}$. The distance between the GDD and LTF reduced the amount of incident light from the GDD to the LTF, thereby limiting the detection level. We have performed the same experiment with a minimum separation of $2 \mathrm{~mm}$ between the GDD and the LTF without the filter. The results were found to be impressive as they can achieve better values for irradiance responsivity, $R e=215.23 \mathrm{MHz} / \mathrm{W}$, and noise equivalent power, $\mathrm{NEP}=3.34 \times 10^{-11} \mathrm{~W} / \sqrt{ } \mathrm{Hz}$. Our future work is to accommodate a filter without a frame so that it will be able to obtain an enhanced detection level by the GDD-LTF combination.

Author Contributions: Conceptualization, A.R.K. and D.R.; methodology, L.K.; software, D.R.; validation, A.A., N.K. and Y.Y.; formal analysis, D.R.; investigation, A.R.K.; resources, A.R.K. and D.R.; data curation, A.R.K. and L.K.; writing-original draft preparation, A.R.K.; writing-review and editing, A.A., N.K. and Y.Y.; visualization, A.R.K.; supervision, A.A. and D.R.; project administration, A.A. All authors have read and agreed to the published version of the manuscript.

Funding: This research received no external funding. 
Acknowledgments: The authors are grateful to the administrative and technical support from the Ariel University, Ariel, Israel and Ben-Gurion University of the Negev, Beer Sheva, Israel.

Conflicts of Interest: The authors declare no conflict of interest.

\section{References}

1. Rogalski, A.; Sizov, F. Terahertz Detectors and Focal Plane Arrays. Opto Electron. Rev. 2011, 19, 346-404. [CrossRef]

2. Siegel, P.H. Terahertz Technology. IEEE Trans. Microw. Theory 2002, 50, 910-928. [CrossRef]

3. Abramovich, A.; Kopeika, N.S.; Rozban, D.; Farber, E. Inexpensive Detector for Terahertz Imaging. Appl. Opt. 2007, 46, 7207-7211. [CrossRef] [PubMed]

4. Rozban, D.; Abramovich, A.; Kopeika, N.S.; Farber, E. Terahertz Detection Mechanism of Inexpensive Sensitive Glow Discharge Detector. Appl. Phys. 2008, 103, 093306-1-093306-4. [CrossRef]

5. Virginia Diodes Inc. Product Datasheet. Available online: https://vadiodes.com/en/products-6/detectors (accessed on 14 June 2021).

6. Alain, M.; John, W.; John, G.A. THz Local Oscillator Source. IEEE Microw. Wirel. Compon. Lett. 2004, 14, $253-255$.

7. Hou, L.; Shi, W. Fast Terahertz Continuous-Wave Detector Based on Weakly Ionized Plasma. IEEE Electron Device Lett. 2012, 33, 1583-1585. [CrossRef]

8. Haj Yahya, A.; Klein, A.; Balal, N.; Borodin, D.; Friedman, A. Comparison between Up-Conversion Detection in Glow-Discharge Detectors and Schottky Diode for MMW/THz High-Power Single Pulse. Appl. Sci. 2021, 11, 4172.

9. Abramovich, A.; Kopeika, N.S.; Rozban, D. THz Polarization Effects on Detection Responsivity of Glow Discharge Detectors (GDD). IEEE Sens. 2009, 9, 1181-1184.

10. Rozban, D.; Aharon, A.; Kopeika, N.S.; Abramovich, A. W-Band Chirp Radar Mock-Up Using a Glow Discharge Detector. IEEE Sens. 2013, 13, 139-145. [CrossRef]

11. Aharon, A.; Rozban, D.; Kopeika, N.S.; Abramovich, A. Heterodyne Detection at $300 \mathrm{GHz}$ Using Neon Indicator Lamp Glow Discharge Detector. Appl. Opt. 2013, 52, 4077-4082. [CrossRef]

12. Aharon, A.; Rozban, D.; Ben-laish, M.; Abramovich, A.; Yitzhaky, Y.; Kopeika, N.S. Ultra-Wideband and Inexpensive Glow Discharge Detector for Millimeter Wave Wireless Communication Based on Up-Conversion to Visual Light. Appl. Opt. 2019, 58, F26-F31. [CrossRef] [PubMed]

13. Abramovich, A.; Kopeika, N.S.; Rozban, D. Design of Inexpensive Diffraction Limited Focal Plane Arrays for MM Wavelength and THz Using Glow Discharge Detector Pixels. Appl. Phys. 2008, 104, 033302. [CrossRef]

14. Rozban, D.; Levanon, A.; Joseph, H.; Aharon, A.; Abramovich, A.; Kopeika, N.S.; Yitzhaky, Y.; Belenky, A.; Yadid-Pecht, O. Inexpensive THz Focal Plane Array Imaging Using Neon Indicator Lamps as Detectors. IEEE Sens. 2011, 11, 1962-1968. [CrossRef]

15. Shilemay, M.; Rozban, D.; Levanon, A.; Yitzhaky, Y.; Kopeika, N.S.; Yadid-Pecht, O.; Abramovich, A. Performance Quantification of a Millimeter-Wavelength Imaging System Based on Inexpensive Glow-Discharge-Detector Focal-Plane Array. Appl. Opt. 2013, 52, C43-C49. [CrossRef]

16. Rozban, D.; Aharon, A.; Levanon, A.; Abramovich, A.; Kopeika, N.S. Switching and Fast Operation of Glow Discharge Detector for Millimeter Wave Focal Plane Array Imaging Systems. IEEE Sens. 2015, 15, 6659-6663. [CrossRef]

17. Aharon, A.; Rozban, D.; Klein, A.; Abramovich, A.; Yitzhaky, Y.; Kopeika, N.S. Detection and Up-Conversion of Three-Dimensional MMW/THz Images to the Visible. Photon. Res. 2016, 4, 306-312. [CrossRef]

18. Rozban, D.; Aharon, A.; Kahana, L.; Abramovich, A.; Yitzhaky, Y.; Altan, H.; Kopeika, N.S. Robust, Sensitive, and Inexpensive 2D Focal Plane Array Upconverting MMW Imaging into the Visible. IEEE Photonics Technol. Lett. 2019, 31, 747-750. [CrossRef]

19. Fan, L.; Junkai, J.; Haiyun, L.; Juan, D.; Feng, L. Preliminary Study of Glow Discharge Plasma on the Static Pressure Measurement. In Proceedings of the 1st Global Power and Propulsion Forum GPPF, Zurich, Switzerland, 16-18 January 2014.

20. Kopeika, N.S. Glow Discharge Detection of Long Wavelength Electromagnetic Radiation: Cascade Ionization Process Internal Signal Gain and Temporal and Spectral Response Properties. IEEE Trans. Plasma Sci. 1978, 6, 139-157. [CrossRef]

21. Kopeika, N.S. On the Mechanism of Glow Discharge Detection of Microwave and Millimeter Wave Radiation. Proc. IEEE 1975, 63, 981-982. [CrossRef]

22. Mavrodineanu, R. Hollow Cathode Discharges. Res. Natl. Bur. Stand. 1984, 89, 143. [CrossRef] [PubMed]

23. Vasil'chenko, S.S.; Serdukov, V.I. Emission Spectrum of Neon as a Frequency Reference for Spectrophotometers. Atmos. Ocean. Opt. 2013, 26, 154-158. [CrossRef]

24. Nighan, W.L. Basic Kinetic Processes in Neon Gas Discharge Displays. IEEE Trans. Electron Devices 1981, 28, 625-630. [CrossRef]

25. Wiesemann, K. A Short Introduction to Plasma Physics. arXiv 2014, arXiv:1404.0509. Available online: http://cds.cern.ch/record/ 1693043/files/arXiv:1404.0509.pdf (accessed on 14 June 2021).

26. ams AG Product Datasheet. LTF Specifications. Available online: https://ams.com/tsl237 (accessed on 14 June 2021).

27. Abramovich, A.; Yitzhaky, Y.; Kopeika, N.S.; Kahana, L.; Rozban, D. Up-conversion of Millimeter Waves to Visible Waves: Inexpensive Focal Plane Array MMW Imaging and Ultra-fast MMW Wireless Communication. In Proceedings of the Terahertz Emitters, Receivers, and Applications XI, Online Only, Bellingham, DC, USA, 24 August-4 September 2020; Volume 11499, p. $114990 U$.

28. Kahana, L.; Rozban, D.; Gihasi, M.; Abramovich, A.; Yitzhaky, Y.; Kopeika, N. Inexpensive Millimeter-Wave Communication Channel Using Glow Discharge Detector and Satellite Dish Antenna. Electronics 2020, 9, 677. [CrossRef] 